\title{
Iron requirements based upon iron absorption tests are poorly predicted by haematological indices in patients with inactive inflammatory bowel disease
}

\author{
Miranda C. E. Lomer ${ }^{1,2}$, William B. Cook ${ }^{3}$, Hamid Jan B. Jan-Mohamed ${ }^{1,4}$, Carol Hutchinson ${ }^{3}$, \\ Ding Yong Liu ${ }^{5}$, Robert C. Hider ${ }^{5}$ and Jonathan J. Powell ${ }^{3 *}$ \\ ${ }^{1}$ Nutritional Sciences Division, King's College London, Franklin-Wilkins Building, London SE1 9NH, UK \\ ${ }^{2}$ Department of Gastroenterology, Guy's and Saint Thomas' NHS Foundation Trust, London, UK \\ ${ }^{3}$ MRC Human Nutrition Research, Elsie Widdowson Laboratory, Fulbourn Road, Cambridge CB1 9NL, UK \\ ${ }^{4}$ School of Health Sciences, Universiti Sains Malaysia, Kelantan, Malaysia \\ ${ }^{5}$ Pharmaceutical Science Division, King's College London, Franklin-Wilkins Building, London SE1 9NH, UK
}

(Submitted 1 December 2010 - Final revision received 25 July 2011 - Accepted 5 August 2011 - First published online 9 December 2011)

\begin{abstract}
Fe deficiency and Fe-deficiency anaemia are common in patients with inflammatory bowel disease (IBD). Traditional clinical markers of Fe status can be skewed in the presence of inflammation, meaning that a patient's Fe status can be misinterpreted. Additionally, Fe absorption is known to be down-regulated in patients with active IBD. However, whether this is the case for quiescent or mildly active disease has not been formally assessed. The present study aimed to investigate the relationship between Fe absorption, Fe requirements and standard haematological indices in IBD patients without active disease. A group of twenty-nine patients with quiescent or mildly active IBD and twenty-eight control subjects undertook an Fe absorption test that measured sequential rises in serum Fe over $4 \mathrm{~h}$ following ingestion of $200 \mathrm{mg}$ ferrous sulphate. At baseline, serum Fe, transferrin saturation, non-transferrin-bound Fe (NTBI), ferritin and soluble transferrin receptor were all measured. Thereafter (30-240 min), only serum Fe and NTBI were measured. Fe absorption did not differ between the two groups $(P=0 \cdot 9$; repeated-measures ANOVA). In control subjects, baseline haematological parameters predicted Fe absorption (i.e. Fe requirements), but this was not the case for patients with IBD. Fe absorption is normal in quiescent or mildly active IBD patients but standard haematological parameters do not accurately predict Fe requirements.
\end{abstract}

Key words: Inflammatory bowel disease: Iron absorption: Iron status: Haematological parameters: Iron deficiency

Fe deficiency (ID) and Fe-deficiency anaemia (IDA) are common in patients with inflammatory bowel disease (IBD; approximately $20-30 \%)^{(1,2)}$. Reasons are multifactorial but protein/blood losses in the gut and low dietary Fe intakes are major drivers ${ }^{(3)}$. Fe absorption is clearly down-regulated in patients with active inflammation due to anaemia of chronic disease $^{(4)}$, but it is not clear whether Fe absorption is altered in patients who are in remission. The absorption of $\mathrm{Fe}$ in patients with quiescent or mildly active IBD compared with healthy controls was first assessed in a pilot study, the results of which were inconclusive ${ }^{(5)}$. The outcome of a more recent study by Chermish et al. implies that the absorption of Fe from ferrous calcium citrate, but not iron bisglycinate, is similar in patients with quiescent Crohn's disease compared with healthy subjects ${ }^{(6)}$. However, Chermish et al.'s study was not designed to compare Fe absorption in patients with IBD and healthy controls.

If $\mathrm{Fe}$ absorption is normal in a cohort of patients with quiescent or mildly active IBD $v$. control subjects, then further analysis can consider the relationship between Fe absorption and standard haematological parameters that are used to predict ID or Fe repletion (IR). Apart from assessing bone marrow stores, which is ethically difficult, Fe absorption probably provides the most sensitive test of Fe requirements (i.e. Fe status $)^{(7)}$. Thus, standard haematological parameters that are used to predict Fe status, and may be perturbed in lowgrade chronic inflammation and/or relapsing-remitting inflammation $^{(8)}$, can be assessed for their predictive value or effectiveness. Hence, in the present study, both Fe absorption and its relationship to haematological parameters have been assessed in patients with IBD and control subjects.

Abbreviations: HBI, Harvey-Bradshaw index; IBD, inflammatory bowel disease; ID, Fe deficiency; IDA, Fe-deficiency anaemia; NTBI, non-transferrinbound Fe; TIBC, total Fe-binding capacity.

* Corresponding author: Dr J. J. Powell, email jonathan.powell@mrc-hnr.cam.ac.uk 
The method of sequential blood sampling following ingestion of ferrous sulphate was used, as this provides a direct and relevant measure of $\mathrm{Fe}$ absorption ${ }^{(9)}$ as opposed to utilisation (e.g. erythrocyte incorporation), which may be independently perturbed in inflammatory conditions. In addition, the method of sequential blood sampling allows non-transferrin-bound Fe (NTBI) to be measured. NTBI has been proposed to occur transiently in serum, following ingestion of therapeutic supplements by Fe-deficient subjects ${ }^{(10,11)}$ and even in some subjects with normal Fe stores ${ }^{(11)}$. The rationale is that the rate of absorption is too great for transferrin to completely bind the incoming $\mathrm{Fe}$, and thus a small proportion binds to albumin or citrate, or even undergoes partial hydrolysis forming polyhydroxy Fe species ${ }^{(12)}$. In such forms (i.e. not bound to transferrin), Fe may be prone to redox cycling and therefore promote oxidative stress within the circulation ${ }^{(13,14)}$. It has been proposed that the antioxidant capacity of the mucosa and the circulation is depleted in $\operatorname{IBD}^{(15-17)}$ such that the formation of NTBI could induce oxidative damage more readily than in control subjects.

The present study aimed to investigate the relationship between Fe absorption, Fe requirements and standard haematological indices in IBD patients without active disease. Additionally, it assessed the formation of circulating NTBI in patients with IBD and controls following ingestion of ferrous sulphate.

\section{Experimental methods}

\section{Participants}

Patients with IBD ( $n$ 29: five with ulcerative colitis and twentyfour with Crohn's disease) were recruited from gastrointestinal outpatient clinics at Guy's and St Thomas' NHS Hospital Trust, London, UK. Control subjects ( $n$ 28) were recruited from a local newspaper advert.

\section{Patients}

Patients were aged 18-65 years and in all cases, IBD was diagnosed by histological and/or radiological criteria. Patients with other chronic diseases, hereditary disorders of Fe metabolism (detected by the assessment of common mutations in the HFE gene using 'WAVE ${ }^{\circledR}$, technology based on denaturing HPLC), pregnant and lactating females and those taking proton-pump inhibitors or Fe therapy/supplements within the previous $28 \mathrm{~d}$ were excluded. Additionally, only patients with inactive or mildly active disease using a Harvey-Bradshaw index (HBI) of less than $8^{(18)}$ were recruited for the study. Patients fulfilling these criteria were invited to participate and then had a blood sample taken to assess Fe status (full blood count, ferritin, serum Fe, soluble transferrin receptor and total serum Fe-binding capacity (TIBC)) and inflammatory status (erythrocyte sedimentation rate and C-reactive protein).

\section{Controls}

An advert was placed in a freely available newspaper distributed predominantly within Greater London. Potential subjects responding to the advert were screened by telephone to exclude anyone with known chronic disease, gastrointestinal disorders, hereditary disorders of Fe metabolism and those taking proton-pump inhibitor medication or Fe therapy/ supplements within the previous $28 \mathrm{~d}$. Pregnant and lactating women were also excluded. Volunteers fulfilling these criteria were invited to participate and then had a blood sample taken to assess Fe status and inflammatory status, as detailed above. Mutations of the HFE gene were also assessed.

The study was conducted according to the guidelines laid down in the Declaration of Helsinki, and all procedures involving human subjects were approved by the St Thomas' Hospital Local Research Ethics Committee (EC03/089). Written informed consent was obtained from all subjects.

\section{Study design}

Recruited subjects were invited to attend St Thomas Hospital, London (UK) for a single $4 \mathrm{~h}$ study appointment at a mutually agreeable time. Subjects were requested not to take any multivitamin or mineral supplements for the week preceding the study and were advised to avoid any Fe-rich foods and tea on the day of their appointment. Subjects were not fasted as it has previously been shown that $\mathrm{Fe}$ absorption from oral ferrous sulphate is similar with or without fasting ${ }^{(10)}$.

Upon arrival, signed consent was obtained and each subject was cannulated in the forearm with a $21 \mathrm{G}$ Venflon Pro cannula (Becton Dickinson Infusion Therapy AB, Helsingborg, Sweden) and a $20 \mathrm{ml}$ baseline blood sample was taken using a plastic $20 \mathrm{ml}$ syringe. The collected blood was divided into aliquots into labelled plain, EDTA and SST ${ }^{\circledR}$ (serum separating tube) vacutainers (Becton Dickinson Vacutainer Systems, Plymouth, UK) for the baseline measures as outlined above. Following collection of the baseline blood sample, subjects were given a single ferrous sulphate capsule $(200 \mathrm{mg} ; 65 \mathrm{mg}$ elemental $\mathrm{Fe}$ ) with a glass of water. Further blood samples $(10 \mathrm{ml})$ were collected at 30,60, 120, 180, 210 and $240 \mathrm{~min}$ post-Fe ingestion to measure serum Fe, TIBC and NTBI.

\section{Laboratory analysis}

Baseline samples for ferritin and C-reactive protein (SST vacutainer) and full blood count (EDTA vacutainer) were analysed by the Clinical Chemistry Laboratory using routine laboratory methods. Samples collected in plain vacutainers for serum Fe, NTBI and TIBC were immediately taken following collection (on ice) to a laboratory where the samples were allowed to clot before being centrifuged at $2500 \mathrm{rpm}$ for $10 \mathrm{~min}$ at $4^{\circ} \mathrm{C}$ (Eppendorf 5804R; Eppendorf, Hamburg, Germany). Following centrifugation, serum was transferred to labelled cryovials, frozen and stored at $-80^{\circ} \mathrm{C}$ in labelled boxes. Samples for serum Fe, TIBC and serum transferrin receptor analysis were analysed by routine methods at the Nutritional Biochemistry Laboratory, MRC Human Nutrition Research, Cambridge. Serum NTBI was analysed using a modified version of the method developed by Singh et al. ${ }^{(19)}$ in the Department of Pharmacy, King's College London, as described previously ${ }^{(20)}$. 


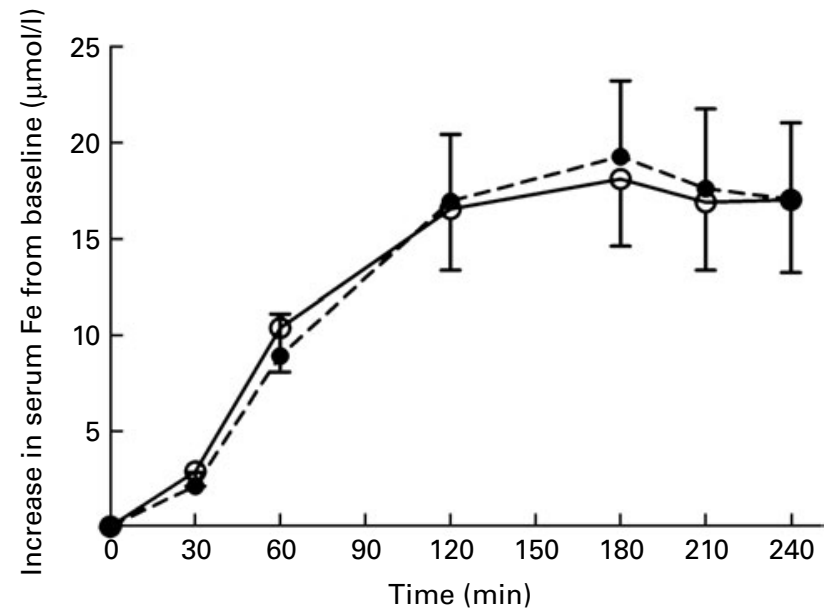

Fig. 1. Increase in serum iron from baseline following ferrous sulphate administration in patients with inflammatory bowel disease $(n 29 ; 0) v$. control subjects $(n 28 ; \bullet) P=0.9$. Values are means, with standard errors of the mean represented by vertical bars.

Transferrin saturation was calculated from TIBC using the following equation:

Transferrin saturation $=100 \times($ serum Fe $/$ TIBC $)$.

\section{Data and statistical analysis}

Subjects with non-Fe-deficiency anaemia, significant inflammation or homozygous mutations of the $H F E$ gene were excluded from the study. Subjects where venous access could not be maintained for the duration of the study were excluded from the analysis. Data were analysed using SPSS version 14 (SPSS, Inc., Chicago, IL, USA) and are presented as means and standard deviations or, where indicated, as means with their standard errors. Unpaired $t$ tests were used to make between-group comparisons of the peak serum $\mathrm{Fe}$ and comparison of baseline markers, of $\mathrm{Fe}$ status and inflammation, between control subjects and patients with IBD. Pearson's product moment correlation coefficient was used to measure the correlation between NTBI, serum $\mathrm{Fe}$ and transferrin saturation. Significance was assumed where $P<0.05$

\section{Results}

A total of seventy-two subjects (IBD $n$ 36, controls $n$ 36) were screened for study recruitment. Of these, five subjects in the control group were not suitable due to non-Fe-related anaemia and two patients with IBD were excluded, one due to moderately active disease $(\mathrm{HBI}=12)$ and the other because he was homozygous for the primary haemochromatosissusceptibility mutation, $\mathrm{C} 282 \mathrm{Y}$. The remaining sixty-five subjects consented to take part but data were incomplete for eight (IBD $n 5$, controls $n$ 3) due to difficulty in maintaining venous access for the duration of the study and were excluded from the analysis. Of the remaining fifty-seven subjects, twenty-eight subjects were controls (twelve males), with a mean age of 35 (SD 11) years and BMI of $23.4(\mathrm{sD} 3) \mathrm{kg} / \mathrm{m}^{2}$, and twenty-nine had IBD (thirteen males), with a mean age of 42 (SD 13) years, BMI of 25.7 (SD 6) $\mathrm{kg} / \mathrm{m}^{2}$ and HBI of 4.2 (SD 1.8). Of these patients with IBD, five had ulcerative colitis and the remaining patients had Crohn's disease (site involved: ileal $n$ 4; ileocaecal $n$ 3; ileocolonic $n$ 8; colonic $n$ 5; site not specified $n$ 4).

Fe absorption was measured by the rise in serum Fe over $4 \mathrm{~h}$, following ingestion of ferrous sulphate, and was similar for patients with IBD ( $n$ 29) and control subjects ( $n$ 28) (Fig. 1). According to the a priori classification of Fe status (see the Methods section), control subjects with ID ( $n$ 10) or IDA $(n 5)$ had peak Fe absorption about $180 \mathrm{~min}$ post-dose and approximately 30 and $45 \mu \mathrm{mol} / 1$ above baseline levels, respectively. In contrast, control subjects without ID (i.e. Fe replete; $n$ 13) had Fe absorption peaks that increased by less than $5 \mu \mathrm{mol} / 1$ (control subjects: IDA or ID $v$. IR $P<0 \cdot 001$; Fig. 2). In patients with IBD and IDA ( $n$ 4), a significant increase in serum Fe levels, peaking at $55 \mu \mathrm{mol} / \mathrm{l}$ above baseline, was observed while in non-anaemic patients with IBD with ID ( $n$ 11) or without ID
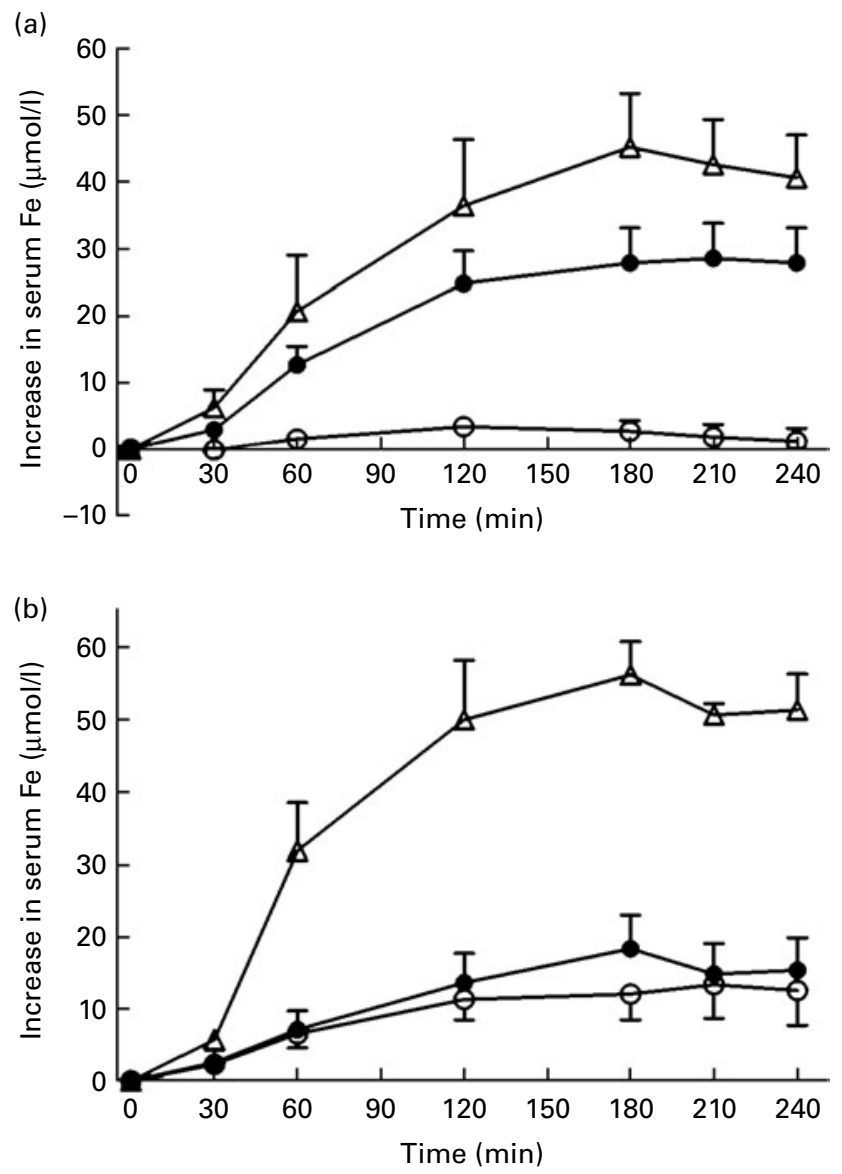

Fig. 2. Increase in serum iron from baseline following ferrous sulphate administration in (a) control subjects ( $n 13$ iron repletion (IR) $O, n 10$ iron deficiency (ID) $\bullet$ and $n 5$ iron-deficiency anaemia (IDA) $\triangle$ ) and (b) patients with inflammatory bowel disease (IBD) ( $n$ 14 IR $\bigcirc, n 11$ ID $\bullet$ and $n 4$ IDA $\triangle$ ) according to the a priori classification of iron status. For patients with IBD, $P=0.8$ for IR $v$. ID and $P<0.05$ for IR $v$. IDA. For control subjects, $P<0.001$ for IR $v$. ID or IDA. Values are means, with standard errors of the mean represented by vertical bars. All according to standard haematological criteria (see the Methods section). 
(i.e. IR; $n$ 14), similar Fe absorption curves were observed with mean peak increases of approximately $15 \mu \mathrm{mol} / 1$ (patients with IBD: ID $v$. IR $P=0 \cdot 8$; Fig. 2 ).

Due to the inability of Fe status markers to predict $\mathrm{Fe}$ absorption, in the absence of anaemia in subjects with IBD (Fig. 2), further assessment was undertaken. The peak rise in serum $\mathrm{Fe}$ is a strong correlate for total $\mathrm{Fe}$ absorption ${ }^{(9)}$. Subjects were thus categorised according to whether they did or did not absorb Fe, and this was related to baseline ferritin, serum $\mathrm{Fe}$ and transferrin saturation in all subjects. Briefly, the Fe status of all subjects was determined using reference ranges for haematological values, so subjects with ID were classified as having (1) serum ferritin $<20 \mu \mathrm{g} / \mathrm{l}$ or (2) serum ferritin $20-55 \mu \mathrm{g} / 1$ and either serum Fe $<14 \mu \mathrm{mol} / 1$ or transferrin saturation $<10 \%^{(21)}$. Mild anaemia was defined as $\mathrm{Hb}$ concentration ranging from 110 to $129 \mathrm{~g} / \mathrm{l}$ for males and 105 to $124 \mathrm{~g} / 1$ for females. Additionally, subjects were also categorised as Fe absorbers or non-absorbers, defined as having a peak serum Fe increase of more than $5 \mu \mathrm{mol} / 1$ or less than $5 \mu \mathrm{mol} / 1$, respectively, following ingestion of $65 \mathrm{mg} \mathrm{Fe}$ as ferrous sulphate. In the control subjects, all four markers (baseline ferritin, serum $\mathrm{Fe}$, transferrin saturation and $\mathrm{Hb}$ ) provided some significant prediction of $\mathrm{Fe}$ requirements (i.e. absorption) as expected, whereas there was no significant predictive power of these markers in patients with IBD (Table 1).

Total Fe absorption, transferrin saturation and NTBI were all strongly correlated for both subject groups (Fig. 3). Surprisingly, even baseline NTBI (i.e. pre-Fe dose) was present in some individuals and, again, correlated with transferrin saturation (Fig. 3). NTBI in the absence of transferrin saturation with $\mathrm{Fe}$ is controversial and, therefore, these data suggest its presence as either an artifact of NTBI assays or an equilibrium product with Fe transferrin, regardless of whether samples come from subjects who are healthy or with IBD. This is further discussed below.

\section{Discussion}

The present findings provide important information on a number of aspects of Fe status and Fe absorption in subjects with IBD.

First, in spite of speculation, Fe absorption is normal in patients with IBD without significant inflammation when compared as a group with healthy controls, and as opposed to patients with active $\mathrm{IBD}^{(5)}$. We recognise that the $\mathrm{HBI}$ is used for assessing clinical activity (i.e. a proxy for inflammation) in patients with Crohn's disease and not with ulcerative colitis, but only five out of twenty-nine had ulcerative colitis in the present study and the findings were the same with all IBD patients ( $n$ 29) or just Crohn's disease patients ( $n$ 24) (data not shown)

Second, IDA in patients with IBD, when assessed by traditional haematological criteria, is predictive of high Fe needs (Fig. 2), whereas ID alone is not (i.e. in the absence of concomitant anaemia) and Fe repletion is not predictive of low Fe needs (Fig. 2). Thus, while patients in this cohort had quiescent or mild IBD and only slight increases in systemic inflammatory markers such as C-reactive protein, erythrocyte sedimentation rate and

Table 1. Comparison of baseline markers of iron status (and inflammation) in control subjects and patients with inflammatory bowel disease (IBD) classified as iron absorbers and non-absorbers ${ }^{*}$

(Mean values and standard deviations, medians and range values)

\begin{tabular}{|c|c|c|c|c|c|c|c|c|c|}
\hline & \multicolumn{4}{|c|}{ Control absorbers ( $n$ 18) } & \multicolumn{4}{|c|}{ Control non-absorbers $(n 10)$} & \multirow[b]{2}{*}{$P$} \\
\hline & Mean & SD & Median & Range & Mean & SD & Median & Range & \\
\hline $\mathrm{Hb}(\mathrm{g} / \mathrm{l})$ & 129 & 12 & 131 & $104-152$ & 148 & 20 & 146 & $130-195$ & 0.005 \\
\hline Ferritin $(\mu \mathrm{g} / \mathrm{l})$ & 25 & 22 & 21 & $3 \cdot 0-90 \cdot 0$ & 99 & 67 & 105 & $15-203$ & $<0.001$ \\
\hline Serum transferrin receptor $(\mathrm{mg} / \mathrm{l})$ & 4.7 & 1.9 & 4.2 & $3 \cdot 1-11 \cdot 0$ & 3.9 & 0.4 & 3.9 & $3 \cdot 2-4 \cdot 7$ & 0.2 \\
\hline Serum Fe $(\mu \mathrm{mol} / \mathrm{l})$ & $12 \cdot 6$ & $5 \cdot 1$ & $11 \cdot 8$ & $4 \cdot 7-22 \cdot 2$ & $20 \cdot 9$ & $7 \cdot 1$ & $18 \cdot 7$ & $11.5-33.0$ & 0.004 \\
\hline $\mathrm{TIBC}(\mu \mathrm{mol} / \mathrm{l})$ & 71 & 10 & 72 & $54.8-91.5$ & 57 & 8 & 53 & $48 \cdot 6-70 \cdot 2$ & $<0.001$ \\
\hline NTBI $(\mu \mathrm{mol} / \mathrm{l})$ & 0.3 & 0.4 & 0.2 & $-0.2-1.5$ & 0.7 & 0.3 & 0.6 & $0.4-1.5$ & 0.005 \\
\hline Transferrin saturation (\%) & $23 \cdot 1$ & 8.2 & $22 \cdot 0$ & $10 \cdot 0-38 \cdot 0$ & $34 \cdot 0$ & $11 \cdot 8$ & $36 \cdot 0$ & $21-50$ & $<0.001$ \\
\hline $\operatorname{ESR}(\mathrm{mm} / \mathrm{h})$ & 5 & 4 & 3 & $1 \cdot 0-13 \cdot 0$ & 2 & 1 & 2 & $2-3 \cdot 0$ & 0.1 \\
\hline CRP (mg/l) & $5 \cdot 1$ & 0.3 & $5 \cdot 0$ & $5 \cdot 0-6 \cdot 0$ & $5 \cdot 8$ & 1.6 & $5 \cdot 0$ & $5 \cdot 0-9 \cdot 0$ & 0.07 \\
\hline \multirow[t]{3}{*}{ Platelets $\left(10^{9}\right)$} & 234 & 45 & 232 & $141-321$ & 216 & 34 & 220 & $168-278$ & 0.3 \\
\hline & \multicolumn{4}{|c|}{ IBD absorbers $(n 21)$} & \multicolumn{4}{|c|}{ IBD non-absorbers ( $n 8)$} & \\
\hline & Mean & SD & Median & Range & Mean & SD & Median & Range & $P$ \\
\hline $\mathrm{Hb}(\mathrm{g} / \mathrm{l})$ & 133 & 15 & 133 & $88-160$ & 140 & 8 & 140 & $130-149$ & 0.3 \\
\hline Ferritin $(\mu \mathrm{g} / \mathrm{l})$ & 39 & 23 & 39 & $5 \cdot 0-94 \cdot 0$ & 39 & 14 & 38 & $22-62$ & 0.7 \\
\hline Serum transferrin receptor $(\mathrm{mg} / \mathrm{l})$ & 4.4 & $1 \cdot 2$ & $4 \cdot 3$ & $2.6-7.5$ & $4 \cdot 1$ & 1.0 & 4.4 & $2 \cdot 0-4.9$ & 0.7 \\
\hline Serum Fe $(\mu \mathrm{mol} / \mathrm{l})$ & $16 \cdot 3$ & $7 \cdot 7$ & $15 \cdot 7$ & $3 \cdot 0-30 \cdot 2$ & 13.9 & $7 \cdot 2$ & $13 \cdot 2$ & $5 \cdot 6-27.9$ & 0.5 \\
\hline $\mathrm{TIBC}(\mu \mathrm{mol} / \mathrm{l})$ & 61 & 10 & 62 & $41 \cdot 0-80 \cdot 0$ & 57 & 10 & 59 & $42 \cdot 1-72 \cdot 5$ & 0.3 \\
\hline NTBI $(\mu \mathrm{mol} / /)$ & 0.4 & 0.3 & 0.3 & $0.0-1.0$ & 0.2 & 0.3 & 0.2 & $-0 \cdot 1-2 \cdot 7$ & 0.3 \\
\hline Transferrin saturation (\%) & $21 \cdot 3$ & 8.5 & $22 \cdot 8$ & $6 \cdot 6-40 \cdot 1$ & $30 \cdot 4$ & $16 \cdot 9$ & $30 \cdot 0$ & $7 \cdot 0-63 \cdot 0$ & 0.2 \\
\hline $\mathrm{ESR}(\mathrm{mm} / \mathrm{h})$ & 14 & 11 & 9 & $5 \cdot 0-42 \cdot 0$ & 10 & 9 & 7 & $2 \cdot 0-26 \cdot 0$ & 0.4 \\
\hline CRP $(\mathrm{mg} / \mathrm{l})$ & 8.4 & $7 \cdot 7$ & $6 \cdot 0$ & $5 \cdot 0-39 \cdot 0$ & $10 \cdot 7$ & 8.6 & 7.0 & $5 \cdot 0-28 \cdot 0$ & 0.5 \\
\hline Platelets $\left(10^{9}\right)$ & 293 & 62 & 290 & $199-457$ & 276 & 64 & 274 & $170-381$ & 0.5 \\
\hline
\end{tabular}

TIBC, total Fe-binding capacity; NTBI, non-transferrin-bound Fe; ESR, erythrocyte sedimentation rate; CRP, C-reactive protein.

* See the Methods section for the categorisation of Fe absorbers and non-absorbers. 

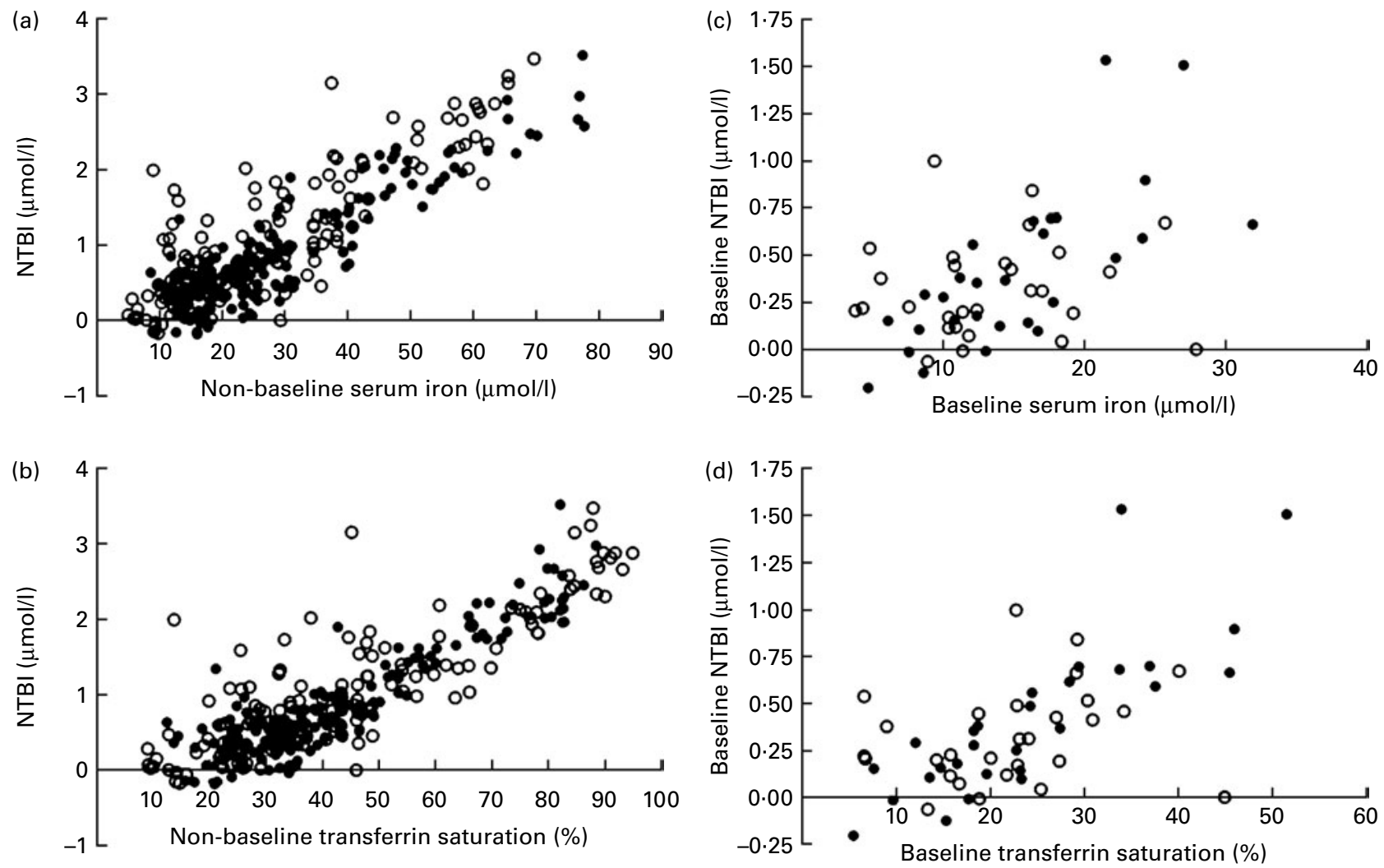

Fig. 3. Correlation between non-transferrin-bound iron (NTBI) and (a) non-baseline serum iron $\left(r^{2} 0.74 ; P<0.001\right)$, (b) non-baseline transferrin saturation $\left(r^{2} 0.77\right.$; $P<0.001)$, (c) baseline serum iron $\left(r^{2} 0.24 ; P<0.001\right)$ and (d) baseline transferrin saturation $\left(r^{2} 0.40 ; P<0.001\right)$ in patients with inflammatory bowel disease (IBD; $n 29 ; O)$ and control subjects $(n 28 ; \bullet) . r^{2}$ and $P$ values are for patients with IBD and control subjects combined. Non-baseline refers to all values that were not at baseline (i.e. all time points post-iron dose).

platelets, this appears sufficient to affect measures based on Fe, transferrin and ferritin as markers of Fe requirements as is well known with more severe inflammation. Levels of serum soluble transferrin receptor have been proposed to be less influenced by inflammation than levels of circulating ferritin or Fe/transferrin $^{(22)}$, but this measure was also insensitive in assessing $\mathrm{Fe}$ needs in IBD. Thus, in the absence of anaemia, current haematological parameters are unable to predict Fe stores, and hence $\mathrm{Fe}$ requirements, even in patients with quiescent or mildly active IBD. Given that oral ferrous Fe supplementation has, on occasions, been associated with severe gastrointestinal adverse effects in IBD $^{(23)}$, as also demonstrated in animal models of colitis ${ }^{(24-26)}$, these data suggest that Fe supplementation should only be undertaken in subjects with ID and IDA where Fe needs are clear. Even then, anaemia of chronic disease, which normally is associated with active IBD, should be ruled out because Fe absorption is then markedly downregulated $^{(5)}$. Diagnostic criteria for the assessment of ID and IDA in IBD in the presence and absence of inflammation have been developed ${ }^{(27)}$.

Third, in agreement with previous work ${ }^{(10,11)}$, the present study has shown that following a single dose of oral ferrous sulphate, there is a rise in apparently detectable NTBI in the circulation of control subjects and in patients with IBD (Figs. 1 and 3). Transferrin is not just an Fe transport protein but is also an anti-redox substrate, so any circulating Fe that is not bound to transferrin (i.e. NTBI) has the capacity for systemic oxidative activity. Individuals with low mucosal and systemic antioxidant status, as has been proposed for patients with IBD $^{(15-17)}$, could, in theory, be especially prone to NTBI effects, including vascular damage and lipid peroxidation $^{(13,14,28)}$. Unsurprisingly, the detection of NTBI in serum was strongly correlated with total Fe uptake into serum and, therefore, the degree of transferrin saturation (Fig. 3). However, unexpectedly, baseline serum Fe levels and transferrin saturation (i.e. fasting and pre-ferrous sulphate) also correlated with NTBI (Fig. 3). This raises the question of whether, outside of the Fe overload, NTBI genuinely exists as a function of partially saturated transferrin or is an artifact of NTBI assays (i.e. do NTBI assays compete for a small fraction of transferrin-bound $\mathrm{Fe}$ in proportion to total serum Fe concentration?). Although beyond the scope of the present study, these issues should be resolved in further work, as it is important to establish whether circulating NTBI really occurs following supplemental oral Fe.

In summary, (1) there was no evidence for Fe absorption to be dysfunctional in patients with IBD. (2) In quiescent or mildly active IBD, IDA clearly indicates a need for $\mathrm{Fe}$ repletion. (3) In the absence of anaemia, standard haematological criteria cannot predict Fe requirements in IBD. (4) A signature for NTBI is detected in patients with IBD to the same extent as it is detected in control subjects, following ingestion 
of ferrous sulphate, but further work is required to determine the authenticity of this apparent NTBI.

Overall, the implications of the present study are that if IBD patients with ID are to be supplemented with Fe, then they should have a concomitant anaemia consistent with IDA (typically a microcytic anaemia). Based on previous work, optimal Fe absorption (i.e. supplemental effectiveness) will be inhibited in patients with active disease ${ }^{(5)}$, while oral Fe may still exacerbate symptoms ${ }^{(23)}$ so the choice between enteral and parenteral Fe supplementation, and which patients to target, should be carefully considered.

\section{Acknowledgements}

We thank Shruti Aggarwal, Juneeshree Shrestha, Ruth Ponting and Hannah Roberts for their help with data collection and analysis. We are grateful to Adrian Mander for help with the statistical analysis. Funding for M. C. E. L. was provided by the PPP Foundation and the DH NHS R\&D Programme, and for H. J. B. J.-M. was provided by the Department of Public Service of Malaysia. W. B. C. was in receipt of an MRC studentship. The authors do not have financial conflicts of interest of any kind, nor have personal relationships with other people or institutions that could inappropriately influence the present study. There is no supplementary online material. The authors' responsibilities were as follows: M. C. E. L. and J. J. P. designed the study. M. C. E. L., W. B. C. and H. J. B. J.-M. carried out the study. D. Y. L. and R. C. H. were responsible for analysing the serum samples for NTBI. M. C. E. L., J. J. P., W. B. C. and C. H. carried out the data analysis. J. J. P. had primary responsibility for the final content of the manuscript. All authors contributed to the preparation of the manuscript and approved the manuscript.

\section{References}

1. Gasche C, Reinisch W, Lochs H, et al. (1994) Anemia in Crohn's disease. Importance of inadequate erythropoietin production and iron deficiency. Dig Dis Sci 39, 1930-1934.

2. Oldenburg B, Koningsberger JC, Berge Henegouwen GP, et al. (2001) Iron and inflammatory bowel disease. Aliment Pharmacol Ther 15, 429-438.

3. Gasche C, Lomer MC, Cavill I, et al. (2004) Iron, anemia, and inflammatory bowel diseases. Gut 53, 1190-1197.

4. Semrin G, Fishman DS, Bousvaros A, et al. (2006) Impaired intestinal iron absorption in Crohn's disease correlates with disease activity and markers of inflammation. Inflamm Bowel Dis 12, 1101-1106.

5. Bartels U, Pedersen NS \& Jarnum S (1978) Iron absorption and serum ferritin in chronic inflammatory bowel disease. Scand J Gastroenterol 13, 649-656.

6. Chermish I, Tamir A, Suissa A, et al. (2006) Ferrous calcium citrate is absorbed better than iron bisglycinate in patients with Crohn's disease, but not in healthy controls. Dig Dis Sci 51, 942-945.

7. Heinrich HC, Bruggemann J, Gabbe EE, et al. (1977) Correlation between diagnostic ${ }^{59} \mathrm{Fe}^{2+}$ absorption and serum ferritin concentration in man. $Z$ Naturforsch C 32, 1023-1025.

8. Thomson AB, Brust R, Ali MA, et al. (1978) Iron deficiency in inflammatory bowel disease. Diagnostic efficacy of serum ferritin. Am J Dig Dis 23, 705-709.
9. Conway RE, Geissler CA, Hider RC, et al. (2006) Serum iron curves can be used to estimate dietary iron bioavailability in humans. J Nutr 136, 1910-1914.

10. Hutchinson C, Al-Ashgar W, Liu DY, et al. (2004) Oral ferrous sulphate leads to a marked increase in pro-oxidant nontransferrin-bound iron. Eur J Clin Invest 34, 782-784.

11. Dresow B, Petersen D, Fischer R, et al. (2008) Non-transferrin bound iron in plasma following administration of oral iron drugs. Biometals 21, 273-276.

12. Hider RC (2002) Nature of nontransferrin-bound iron. Eur J Clin Invest 32, Suppl. 1, 50-54.

13. Cighetti G, Duca L, Bortone L, et al. (2002) Oxidative status and malondialdehyde in beta-thalassaemia patients. Eur $J$ Clin Invest 32, Suppl. 1, 55-60.

14. Rooyakkers TM, Stroes ES, Kooistra MP, et al. (2002) Ferric saccharate induces oxygen radical stress and endothelial dysfunction in vivo. Eur J Clin Invest 32, Suppl. 1, 9-16.

15. Buffinton GD \& Doe WF (1995) Depleted mucosal antioxidant defenses in inflammatory bowel disease. Free Radic Biol Med 19, 911-918.

16. Koutroubakis IE, Malliaraki N, Dimoulios PD, et al. (2004) Decreased total and corrected antioxidant capacity in patients with inflammatory bowel disease. Dig Dis Sci 49, 1433-1437.

17. Maor I, Rainis T, Lanir A, et al. (2008) Oxidative stress, inflammation and neutrophil superoxide release in patients with Crohn's disease: distinction between active and nonactive disease. Dig Dis Sci 53, 2208-2014.

18. Harvey RF \& Bradshaw JM (1980) A simple index of Crohn's disease activity. Lancet 315, 514.

19. Singh S, Hider RC \& Porter JB (1990) A direct method for quantification of non-transferrin-bound iron. Anal Biochem 186, 320-323.

20. Kooistra MP, Kersting S, Gosriwatana S, et al. (2002) Nontransferrin bound iron in the plasma of haemodialysis patients after intravenous iron saccharate infusion. Eur $J$ Clin Invest 32, Suppl. 1, 36-41.

21. Gasche C, Dejaco C, Waldhoer T, et al. (1997) Intravenous iron and erythropoietin for anemia associated with Crohn disease. A randomized, controlled trial. Ann Intern Med 126, 782-787.

22. Jayaranee S \& Sthaneshwar P (2006) Serum soluble transferrin receptor in hypochromic microcytic anemia. Singapore Med J 47, 138-142.

23. Schroder O, Mickisch O, Seidler U, et al. (2005) Intravenous iron sucrose versus oral iron supplementation for the treatment of iron deficiency anemia in patients with inflammatory bowel disease - a randomized, controlled, open-label, multicenter study. Am J Gastroenterol 100, 2503-2509.

24. Carrier J, Aghdassi E, Jeejeebhoy K, et al. (2006) Exacerbation of dextran sulfate sodium-induced colitis by dietary iron supplementation: role of NF-кB. Int J Colorectal Dis 21, 381-387.

25. Erichsen K, Milde AM, Arslan G, et al. (2005) Low-dose oral ferrous fumarate aggravated intestinal inflammation in rats with DSS-induced colitis. Inflamm Bowel Dis 11, 744-748.

26. Seril DN, Liao J, Yang CS, et al. (2005) Systemic iron supplementation replenishes iron stores without enhancing colon carcinogenesis in murine models of ulcerative colitis: comparison with iron-enriched diet. Dig Dis Sci 50, 696-707.

27. Gasche C, Berstad A, Befrits R, et al. (2007) Guidelines on the diagnosis and management of iron deficiency and anemia in inflammatory bowel diseases. Inflamm Bowel Dis 13, 1545-1553.

28. van Tits LJ, Jacobs EM, Swinkels DW, et al. (2006) Serum non-transferrin-bound iron and low-density lipoprotein oxidation in heterozygous hemochromatosis. Biochem Biophys Res Commun 345, 371-376. 